\title{
Formation of size-tunable and nearly monodisperse InP nanocrystals: chemical reactions and controlled synthesis
}

Zheheng Xu, Yang Li, Jiongzhao Li, Chaodan Pu, Jianhai Zhou, Liulin Lv, Xiaogang Peng*

Center for Chemistry of Novel \& High-Performance Materials, Department of Chemistry, Zhejiang University, Hangzhou 310027, People's Republic of China

*Correspondence to: xpeng@ @ju.edu.cn (XP).
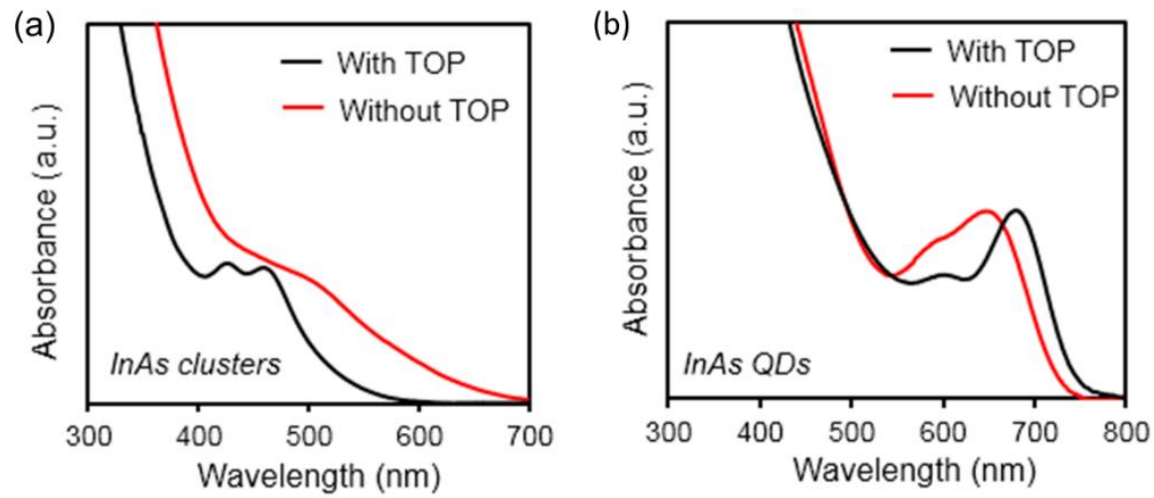

Figure S1. UV-vis spectra of InAs clusters (a) synthesized with and without TOP and QDs (b) formed with two types of InAs clusters shown in (a).

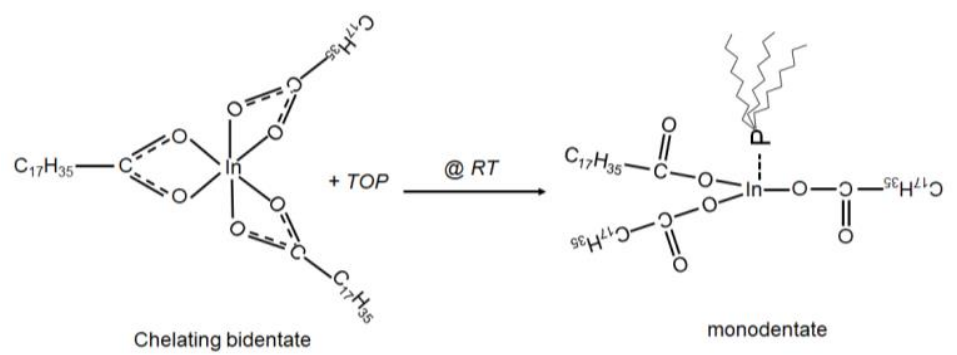

Figure S2. The configuration transform of indium stearate after being coordinated with TOP, from octahedron to tetrahedron.
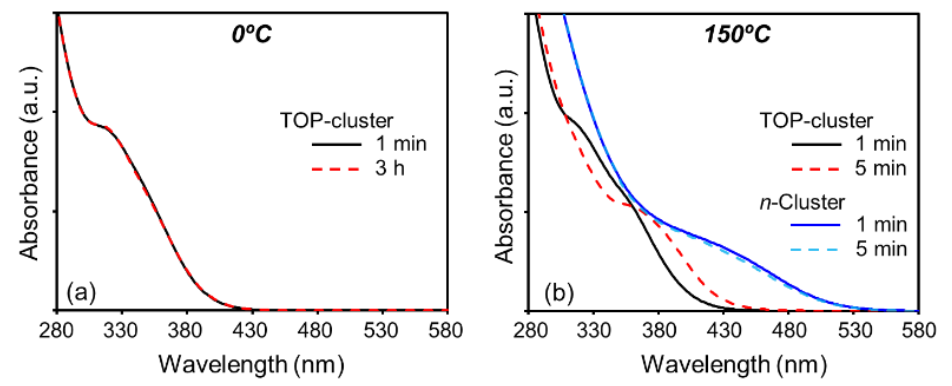

Figure S3. Temporal evolution of the UV-vis spectra of TOP-cluster at $0^{\circ} \mathrm{C}$ (a) and $150^{\circ} \mathrm{C}$ (b). For comparison, absorption spectra of $n$-Clusters are given in (b). 


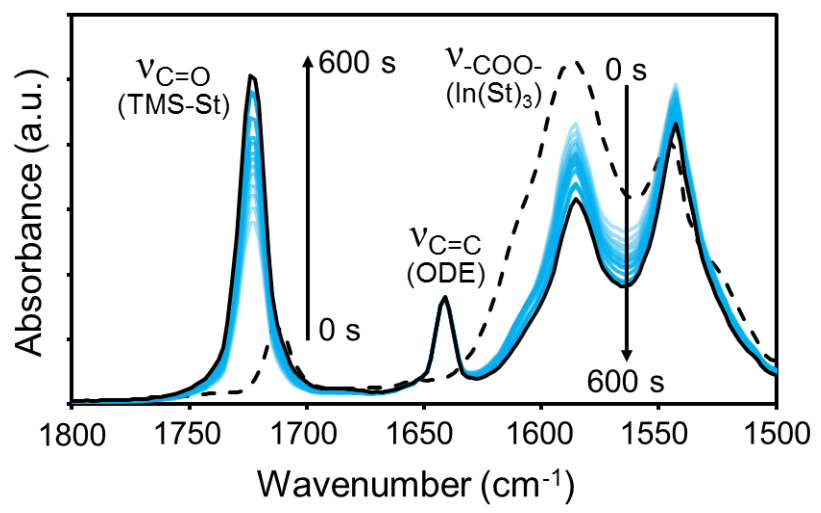

Figure S4. Temporal evolution of the in-situ FTIR spectra during formation of $n$-Cluster at room temperature.
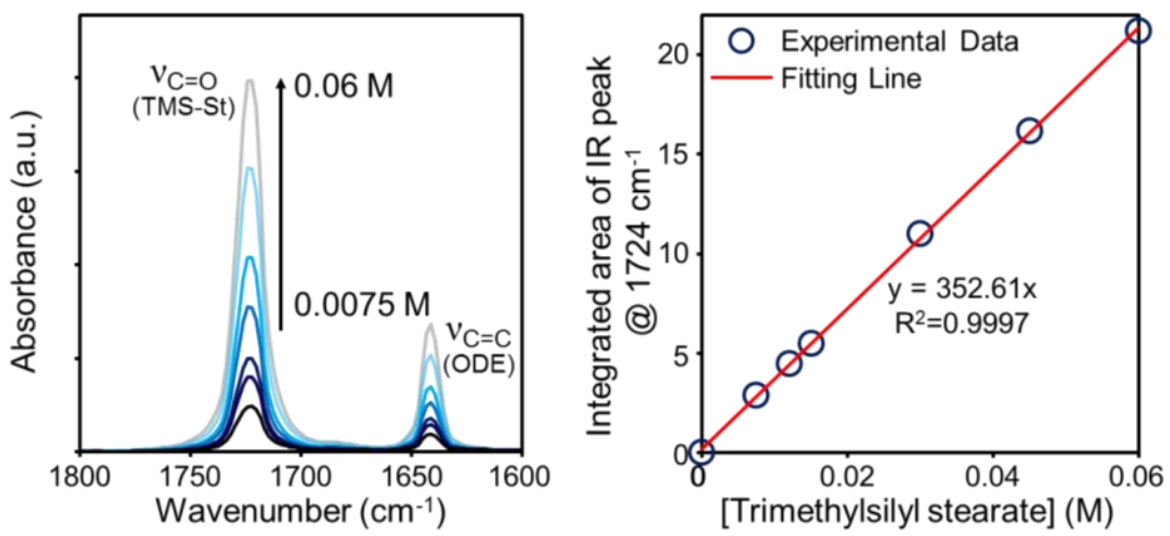

Figure S5. FTIR calibration curve for carbonyl group of trimethylsilyl stearate, dissolved in ndodecane (optical path $600 \mu \mathrm{m})$. With the long optical path $(600 \mu \mathrm{m})$ cuvette, the curve features a perfect linear relationship between the peak area of the carbonyl vibration and concentration of trimethylsilyl stearate. An excess amount of the P precursor was allowed to react with a fixed concentration of indium stearate. Then the resulting products was diluted to the target concentration for the measurements.

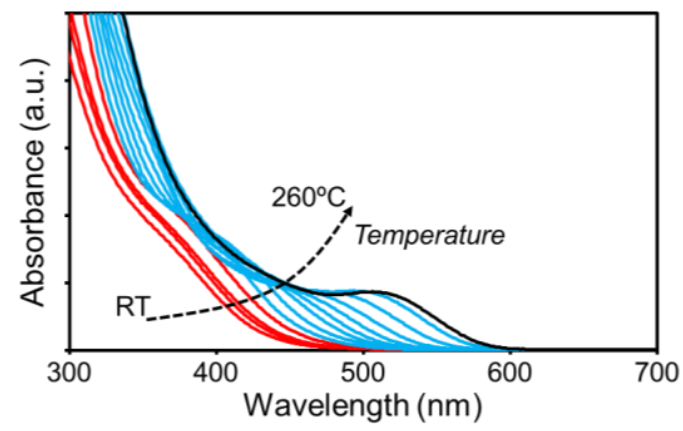

Figure S6. Temporal evolution of UV-Vis spectra for a heating-up reaction of the pre-formed $n$ Cluster 50 during the heating-up stage (red curves), at $260^{\circ} \mathrm{C}$ (blue curves), and the final product (black curve) 

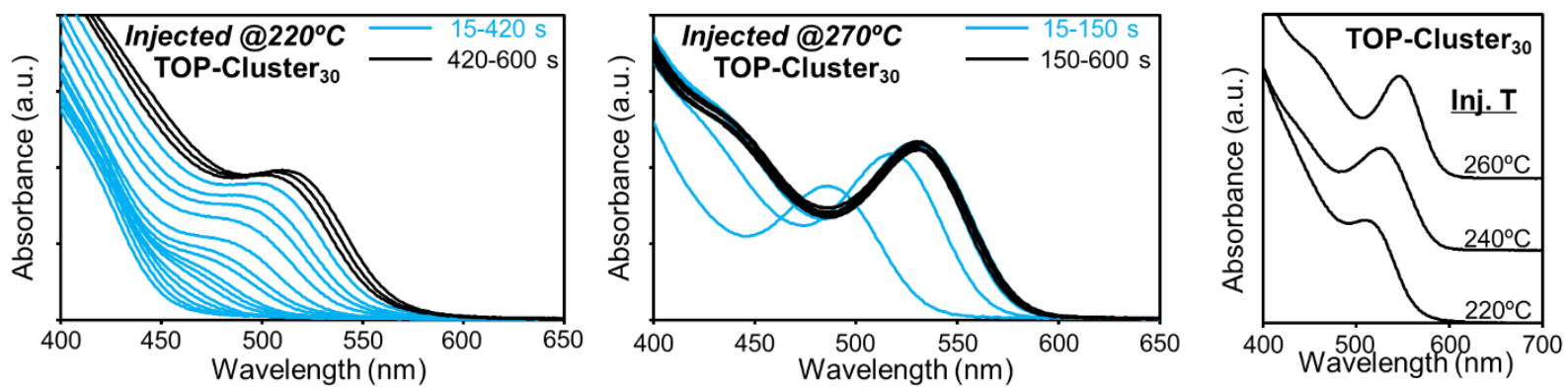

Figure S7. Temporal evolution of the UV-vis spectra for formation of InP QDs by injection of TOP-Cluster 30 at $220^{\circ} \mathrm{C}$ (left) and $270^{\circ} \mathrm{C}$ (middle). (right) The UV-vis spectra of the final product by injecting TOP-cluster 30 at different temperatures.

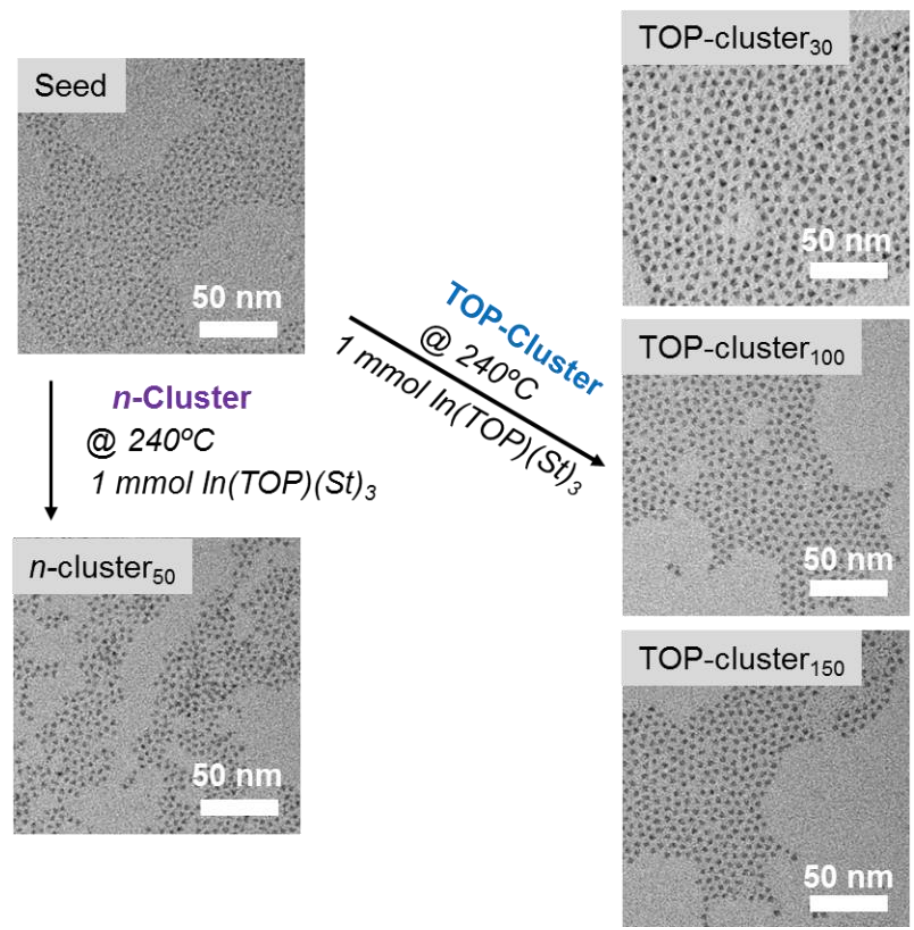

Figure S8. TEM images corresponding to the samples in Figure $4 \mathrm{~b}$. 

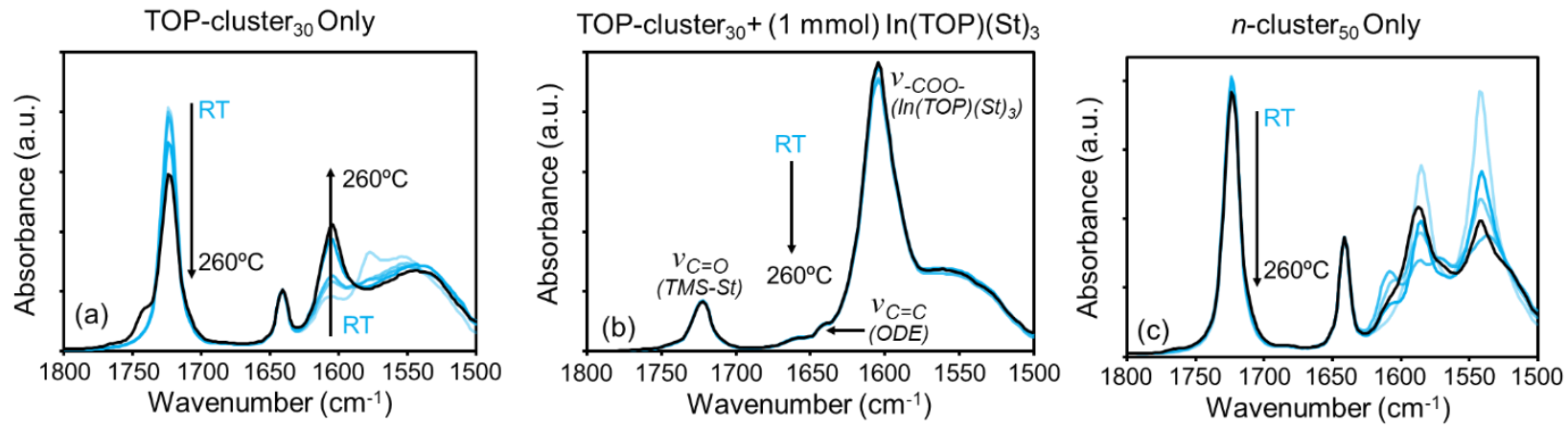

Figure S9. Evolution of FTIR spectra of the reaction mixture by heating up from room temperature to $260^{\circ} \mathrm{C}$ (a) pre-formed TOP-cluster 30 and (b) pre-formed TOP-cluster 30 plus a large excess of $\operatorname{In}(\mathrm{TOP})(\mathrm{St})_{3}$. (c) Evolution of FTIR spectra of the reaction mixture by heating up pre-formed $n$ cluster 50 from room temperature to $260^{\circ} \mathrm{C}$.

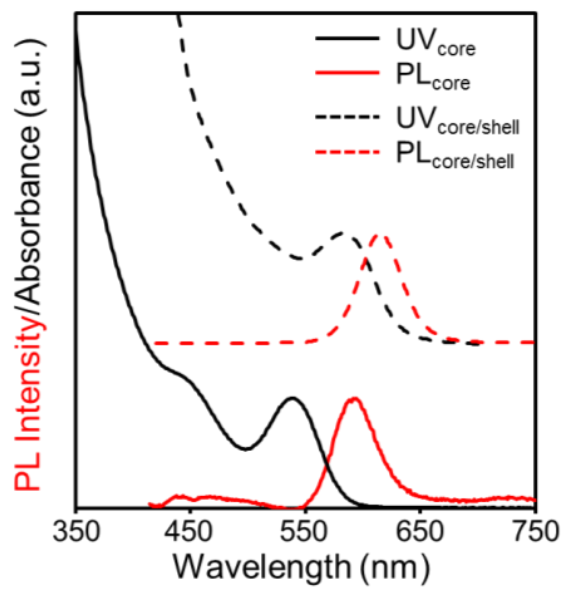

Figure S10. UV-Vis and photoluminescence (PL) spectra of InP core and InP/ZnSe core/shell QDs. FWHM of the PL of the core/shell QDs is $40 \mathrm{~nm}$ without extensive optimization. 

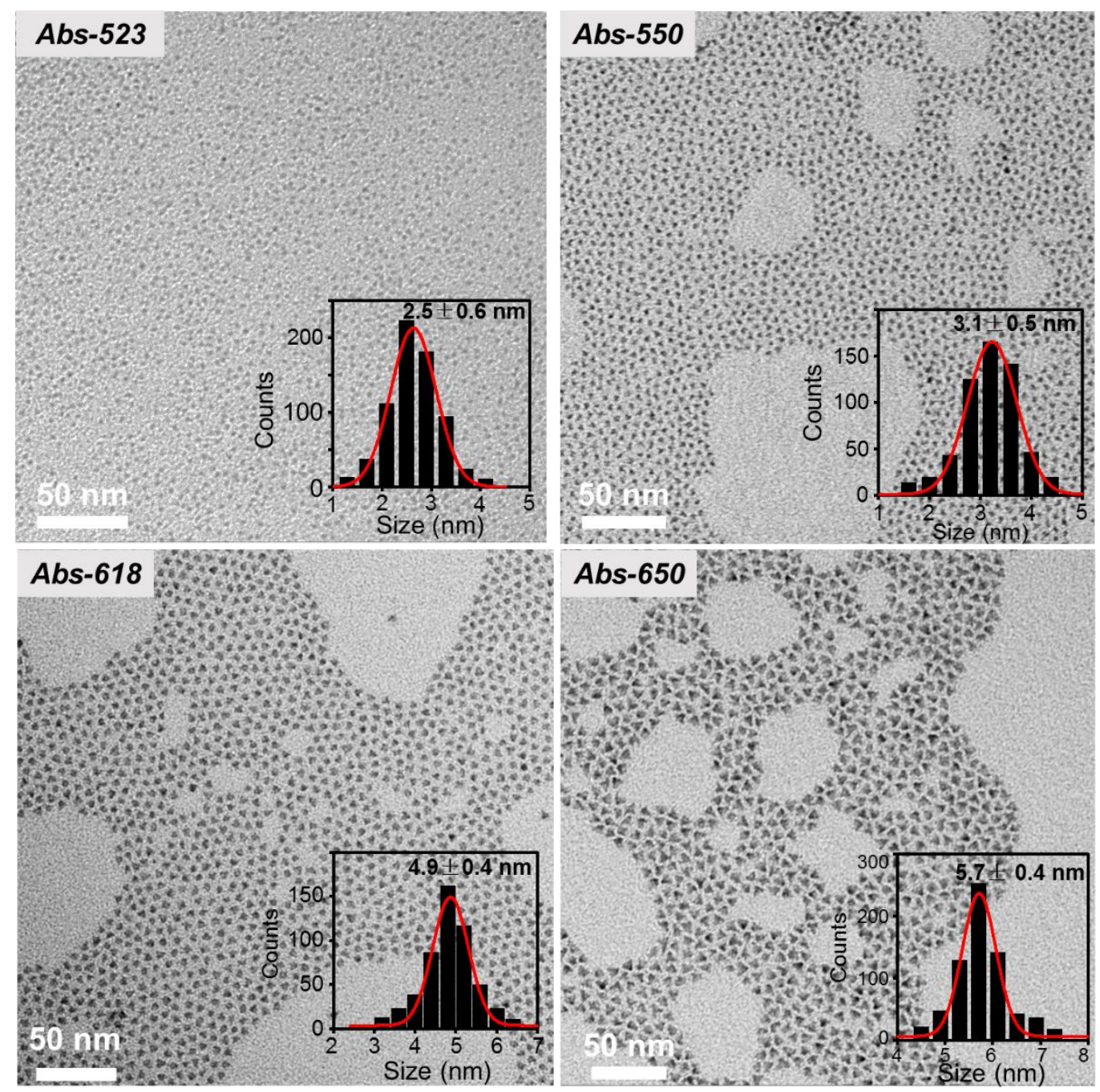

Figure S11. Typical TEM images and size distribution histograms of InP nanocrystals in Figure 6a. 